\title{
How to improve the technological innovation capability of latecomer firms An integrated perspective
}

\author{
Lei Yang ${ }^{1, a}$, Haibing Liu ${ }^{2, b *}$ \\ ${ }^{1}$ School of Economics and Management, Lanzhou Jiaotong University, Lanzhou, China \\ ${ }^{2}$ School of Economics and Management, Lanzhou Jiaotong University, Lanzhou, China
}

\begin{abstract}
This paper discusses the evolution track and micro-mechanism of LCFs' technological innovation capability from the perspective of integration of "Innovation Strategy-Innovation Paradigmtechnological Innovation capability(SPT)". It makes an longitudinal exploratory case of Huawei Company from 1987 to 2018. In this paper, it is found that: first, Huawei's evolution of technological innovation capability is "Imitation Innovation capability-Primary Secondary Innovation capability - Ripe Secondary Innovation capability - Integrated Innovation capability - Original Innovation capability". Technology innovation capability can be measured from three dimensions of technical distance, technical efficiency and technical reserves. Second, absorptive capability is the intermediate variable between the interaction of innovation strategy and technological innovation capability. Third, the driving mechanism, regulating mechanism and catalytic mechanism among the integrated evolution model in SPT of LCFs' are respectively the Knowledge Cycle Mechanism, Knowledge Diversity and Dissipative Structure Theory. The theoretical contribution of this paper is as follows: it provides a new theoretical perspective for the followup research, and also provides a theoretical reference for the existing LCFs to get out of the bottleneck of development.
\end{abstract}

\section{Introduction}

Latecomer Firms(LCFs) is an independent concept different from Late Entrant and Start-up (Mathews \& Cho, 1999). Since the Reform and Opening, the rise of Chinese manufacturing latecomer firms, represented by Huawei, has aroused widespread concern all over the world. However, with the gradual embodiment of the advantages of latecomer, the technology leaders began to export technology defensively, and the "ceiling effect" gradually became the main problem in the development of latecomer firms. How to establish an efficient catchup mode, break the existing technological shackles, and realize "beyond-catch-up" is becoming an urgent problem to be solved in the latecomer firms of our country.

At present, the research on the catch-up of latecomer firms has become very rich, focusing on the Technology Learning path of catching up in latecomer firms, influencing factors of innovation performance of latecomer enterprises under the situation of catching up, Catch-up Strategy of latecomer firms. Chen Litian(2012) carried out rich research on the evolution path of technological innovation capability, and deduced the path of technological innovation capability in combination of absorption, integration and original innovation capability; Liu Haibing\&Xu QingRui(2018) creatively constructed an integrated theoretical model based on the relationship between strategy, innovation and ability, and emphasized the leading role of strategy in the process of innovation paradigm and ability evolution of latecomer enterprises.

However, there are still the following theoretical gaps in the existing research: first of all, there are few literature focusing on the evolution of technological innovation capability of latecomer firms, and there is a lack of more accurate measurement of technological innovation capability. Secondly, the evolution of the ability of latecomer firms comes from the active choice of enterprises, or passive adaptation to this problem has always been a blind spot in the field of innovation management (Xu Qingrui et al., 2013).

To sum up, none of the above studies focus on the capability of technological innovation. From the perspective of "innovation strategy - innovation paradigm - technological innovation capability", this paper discusses the micro-mechanism of realizing efficient catch-up in latecomer firms, which is the main topic of this paper.

ayangleilei2588@163.com; * Corresponding author: bhabliu@zju.edu.cn

Haibing Liu is correspondence author of this paper. 


\section{Theory}

\subsection{Technological Innovation capability}

The concept of technological innovation capability began at the end of last century and is the expansion of technological innovation concept from the perspective of capability. After that, the research mainly focuses on the interactive relationship between technological innovation and innovation strategy, the process of technological innovation (Larry,1981), the perspective of resource flow in technological innovation, the enterprise technology source model (Sher,2005) and so on. The research is carried out from different theoretical bases.

Research on the path evolution of technological innovation capability, Qingrui Xu(2013) described the evolution path of enterprise independent innovation capability in transitional economy as "secondary innovation capability-integrated innovation capabilityoriginal innovation capability". Chen Litian (2012) defines the different stages of the development of technological ability by the combination of absorption, integration and originality, but the above research lacks the accurate measurement of technological innovation capability.

As for the measurement of technological innovation capability, most of the existing research is based on the systematicness of technological innovation capability, and measures the whole process of technological innovation. After that, some scholars believe that the above measurement index are lack of theoretical hierarchy, and are too similar to the measurement of enterprise ability.

Based on the specific situation of technological catch-up in latecomer firms, combined with the technology theory of Fransman (1984), this study combs the measurement index of technological innovation capability of Qu Guoyu and Liu Xueming (1999), and select three measurement dimensions: technical distance, technical efficiency and technical reserve. The technical distance can reflect the position of the latecomer in the industry technology system. Technical efficiency and technology reserve can depict the linear or nonlinear evolution of technology system in latecomer firms. Through the different combinations of the above three measurement dimensions, the types of technological innovation capability can realize an improvement from quantitative to qualitative.

\subsection{Innovation Strategy Of Latecomer Firms}

Latecomer Firms(LCFs) is widely recognized as a developing country enterprise which faces both technical and market competitive disadvantages, but at the same time has the advantage of latecomer. The existing research on latecomer firms mainly focuses on catching up opportunity window, low cost advantage(Mathews, 2002), increasing local market space and so on. Latecomer firms are also an important situational theory in the study of the development of Chinese enterprises.
Benner(2002) divide enterprise strategy into exploratory strategy and exploitative strategy according to the utilization of innovation resources. Wu Xiaobo et al(2019) are based on the situation of latecomer firms. The innovation strategy of latecomer firms is divided into four types: market exploratory, market exploitative, technology exploratory and technology exploitative. This paper uses its index system to investigate the case data.

\subsection{Innovation Paradigm}

Innovation paradigm is a concept that includes, but is not limited to, innovation norms and innovation models (Liu Haibing, 2018) The research on the classification of innovation paradigm mainly focuses on the following three kinds: progressive innovation and subversive innovation, open innovation and closed innovation, and single innovation, innovation portfolio and total innovation. Based on the research problems, this paper chooses the total innovation paradigm as the research perspective, referring to the investigation of the enterprise innovation model in the book "Total Innovation Management" by academician Xu Qingrui. This paper analyzes the innovation mode of case enterprises from four dimensions: organizational innovation, institutional innovation, management innovation and cultural innovation.

\subsection{Research on the Integration of Innovation Strategy, Innovation Paradigm and Technological Innovation capability of Latecomer firms}

The research on the catch-up mechanism of latecomer firms is very rich: the research from the micro perspective mainly focuses on the technical level, and the macro perspective mainly focuses on different ownership, the perspective of collaborative innovation and dynamic ability evolution; Liu Haibing (2018) constructed an integrated LSIC model of strategic evolution, innovation paradigm and capability evolution of latecomer firms.

However, none of the above studies have deeply discussed the operation mechanism of the integrated model of "innovation strategy - innovation paradigm technological innovation capability" of latecomer firms, which is the problem to be solved in this paper.

\section{Research methods and data sources}

Case study belongs to the category of qualitative research, and a new explanation of the research problem is found through the in-depth description and analysis of typical cases. This paper selects the longitudinal single case exploratory research method to carry on the research: first, the vertical case design can depict the stage characteristic of the technological innovation capability more clearly; Secondly, the case study is more helpful to carry on the anthropological "deep description" to the research object, fully examines the problems in the research framework and carries on the 
thorough discussion, in order to ensure the internal validity of the research conclusion.

\subsection{The Basis Of Case Selection}

The selection of case enterprises should be extreme and typical in order to grasp the main aspects of the research problems. Combined with the research problems, the case enterprises in this paper should have the following characteristics: (1) they belong to the latecomer enterprises, which have experienced the development process from "catching up" to "beyond-catching up", (2) the catch-up mode is typical and the industry is representative; (3) availability of rich information and data.

Based on the above standards, this paper selects Shenzhen Huawei Technology Co. Ltd. (here in after referred to as "Huawei") as the sample enterprise for the following reasons: first, Huawei Company has jumped from the agent with backward technology to first-class enterprise, and the industry influence has been continuously improved, which is one of the few postdevelopment enterprises realizing "beyond catching-up", which is typical. Secondly, Huawei's catch-up history has obvious stage characteristics, which is helpful to ensure the expansibility and saturation of the theory. Finally, the data acquisition is convenient, and the team accumulated a lot of research results in the cooperation with Huawei, and the academic research and news materials of Huawei focus at home and abroad are quite extensive. To sum up, Huawei is selected as the sample enterprise of this study.

\subsection{Data Sources}

Zhejiang University Innovation Management Team has accumulated a large number of first-hand data in the cooperation with Huawei, forming a wealth of research results, such as the monograph and so on. On the basis of combing the internal research results, this paper combines a large number of second-hand data to carry out the research. The second-hand materials mainly come from the internal and external materials of the enterprise: the internal materials are: Huawei annual report, Huawei people's magazine, Huawei official website related reports; Up to May 2, 2019, this paper obtained 1058, 74, 2344 materials are obtained from the database of CNKI, the database of Weipu Journal and the database of Wanfang, which use "Huawei Innovation" as the key word to carry out advanced search; In the global case discovery system innovation and entrepreneurship section, "Huawei" is used as the key word to search, and 16 related materials are obtained.The annual report of Huawei from 06 to 18 is sorted out to find the evidence related to this study.

In this paper, the Huawei historical event library is sorted out by means of open coding, principal axis coding and selective coding, and the pursuit process of Huawei is divided into six stages.

\section{Case analysis}

In this paper, Huawei's development is roughly outlined in the following six stages:

\subsection{Stage I General Agency Period (1987-1990)}

In 1987, Huawei entered the exchange market through agents, established a relatively perfect sales system, implemented a highly centralized decision-making mechanism, and praised the offensive culture.

\subsection{Stage II SPC exchange Age's Technology Innovation capability(1990-1993)}

In 1990, Huawei used a keen sense of market to realize the strategic transformation from agent to manufacturer. At this stage, its strategy is manifested as the market exploitative strategy. At the same time, Huawei adjusts the innovation paradigm to meet the requirements for rapid learning technology. Under the simple linear organizational structure, it accumulates R\&D complementary assets through the market occupation strategy of the "aardwolf", reflecting its "technologydriven" innovation paradigm. Thanks to the absorptive capacity mechanism of "learning by doing", Huawei's technological innovation capability has been accumulated, but it is still limited to the simple imitation of "pioneers", which is manifested in the primary ability of imitation and innovation.

\subsection{Stage III 2G Period: Preliminary Secondary Innovation capability (1993-1997)}

Since 1993, Huawei has gained insight into the huge business opportunities in the mobile communications market and moved to the $2 \mathrm{G}$ market. Huawei first occupied the rural market while increasing $R \& D$ investment, the innovation strategy showed mature market exploitative and low level of technology exploitative. At the same time, Huawei has made adaptive changes to its innovation paradigm through organizational structure adjustment and advocating" overtime culture". The core connotation of the innovation paradigm is "technological catch-up". Through learning by R\&D, Huawei has realized the primitive accumulation of key technology and market knowledge (Xu Qingrui,2013) and entered the second innovation. In the primary development stage $(\mathrm{Wu}$ Xiaobo,2008), the technological innovation capability is the preliminary secondary innovation capability.

\subsection{Stage IV 3G Period: Mature Secondary Innovation capability (1997-2007)}

During the $3 \mathrm{G}$ period, in order to solve the problems of over capability and low efficiency of internal innovation, Huawei established a primary market exploratory and mature technology exploitative innovation strategy. Based on the strategic guidance, Huawei began to reform the technology research and development process, transplant the international standard management model 
and so on. Through the coordination among the various elements, Huawei constructed a "technology-market dual-drive" innovation paradigm. At the same time, Huawei's technological innovation is mainly manifested in the "improved innovation" which focuses on reverse engineering, and realizes the "path jump" from "path following" through "functional understanding" of technology by "learning by using" and "learning by spillovers". Second innovation capability entry to the stage of maturity

\subsection{Stage V: LTE Integrated Innovation capability (2007-2013)}

In order to cope with the defensive technology output of international giants, Huawei has established an innovative strategy of high level and balanced development of market exploration and technology exploitative. At this stage, Huawei adjusted to a moderately decentralized organizational structure, innovatively launched the rotating CEO management mechanism, and advocated customer-oriented corporate culture. The innovation paradigm presented the core connotation of "market pull". Through the "interactive middle school", Huawei already has the capability to explore the core structure of the technology paradigm, begins the nonlinear evolution process of the technology system and enters the stage of "self-oriented" integrated innovation capability.

\subsection{Stage VI 5G: Original Innovation Based Technological Innovation capability (2013- 2019)}

Since 2013, global industrial transformation and crossindustry integration have become the normal. Huawei has put forward an innovation strategy focusing on "market exploration and technology exploration." Under the penetration of this innovative strategy, Huawei's organizational structure has been adjusted to a strong matrix structure, the flexibility of theoretical organization has been enhanced, and the system has been adjusted to take the project as the center. At the same time, it has created an open, cooperative and win-win corporate culture atmosphere. The innovation paradigm presents the core connotation of "market beyond catching-up" (Haibing Liu,2018). Through the " learning by advances in science and technology", Huawei realizes the "change of track" of technology. The "Gestalt" of its technological paradigm has changed and continues to explore on the new technology track. That is, its technological innovation capability developed into the original innovation capability stage.

\section{Results and discussion}

\subsection{Evolution path and Internal Logic of technological Innovation capability}

Focusing on the point of "technological paradigm-technological trajectory", this paper introduces the dimension of technology theory to explore the evolution path of technological innovation capability in a narrow sense and the microscopic mechanism of its evolution.

In the stage of market exploitative strategy in the early stage of production and manufacturing, the primary dimension of Huawei's technological innovation capability is its ability to overcome technical barriers and narrow the "technical distance". The technical efficiency and technical reserve ability lacks the necessary expression environment, which has not been reflected at this stage. Product manufacturing is mainly through the imitation of the forerunner, so this stage is called imitation innovation capability.

In the stage of innovation strategy of high market exploitative and low technology exploitative, Huawei has a low level of technology reserve, but its technical efficiency ability is not obvious. Enterprises still focus on technological catch-up, focusing on narrowing the technological distance through knowledge absorption and domestication. Therefore, Huawei's technology evolution path is still followed by technology, but it has the ability to improve the "outer structure" of the technology paradigm, which is manifested as a preliminary secondary innovation capability.

After Huawei enters the innovation strategy stage with low market exploration and high technology exploitative as the core, the technology distance is obviously reduced, and the technology reserve ability is improved. However, through the introduction of IPD and other R\&D processes, the efficient connection of basic R\&D activities is guaranteed, and the technical efficiency ability is greatly broken. Therefore, Huawei can achieve "path jump" during this period, which is manifested as ripe secondary innovation capability.

In the next development, Huawei implements the strategy of exploratory innovation with high market and low technology. At this stage, there is almost no technical distance, and the phenomenon of "beyondcatch-up" begins to appear. The technical efficiency ability realizes the new accumulation, but the technology pre-research and other strategic measures make the technology reserve ability become the most critical ability representation. Huawei makes use of the lower transfer barrier "detour overtaking" to enter the stage of integrated innovation capability.

With the continuous improvement of the status of the industry, Huawei implements high market exploration and technology exploratory innovation strategy. At this stage, Huawei's technology development has been synchronized or even surpassed with the first-class enterprises, and the technical efficiency has been further improved. At the same time, the technology reserve ability is still represented the core competence. Huawei's "changing track" to 5G technology has leapt to independent innovation capability.

In the above evolution process, the core connotation of Huawei's technological innovation capability has realized the evolution path from "the regulation and control ability of technology distance" to "technology efficiency improvement ability" and then to "technology reserve ability".

The inherent logic of its evolution is the spiral increase of knowledge creation and knowledge 
absorption, which can also explain the reason why some enterprises fall into the "Matthew effect".

\subsection{Strategy, Innovation Paradigm and technological Innovation capability of latecomer Enterprises: an Integrated Model}

This paper summarizes the growth process of Huawei as follows: the evolution path of Huawei's innovation strategy is as follows: "market exploitative -- high market exploitative and low technology exploitative -high market exploration, high technology exploitative -high market exploration and low technology exploration -- high market exploration and technology exploration"; At the same time, the co-evolution path of innovation paradigm is "technology-driven - technology catch-up technology and market parallel drive - market pull market beyond catch-up", which accords with Liu Haibing's conclusions on the evolution of innovation paradigm of latecomer enterprises. The evolution path of technological innovation capability is "imitation innovation capability - primary secondary innovation capability - ripe secondary innovation capability integrated innovation capability - Original innovation capability".

Throughout the evolution of Huawei's catch-up model, it can be found that: strategy leads innovation paradigm, innovation paradigm is the supporting mechanism of strategy implementation, and the coordination among the elements of innovation paradigm plays a key role in improving technological innovation capability (Liu Haibing, 2018). To sum up, the innovation strategy, innovation paradigm and technological innovation capability of latecomer enterprises are a coordinated and inseparable integrated evolution model.

\subsection{Intrinsic mechanism of post-development enterprise strategy, innovation model and technical innovation capability:based on ecological perspective.}

Through the above analysis, this paper abstracts the driving mechanism, regulating mechanism and catalytic mechanism of SPT integrated evolution model from the ecological point of view in the process of catching up with latecomer enterprises, as shown in Fig. 1:

First, the "material" cycle mechanism: under the drive of the situation, the latecomer enterprise absorbs the external knowledge, the knowledge flows from the enterprise to the market, and then feedback to the enterprise through the market, the enterprise adjusts the innovation paradigm to adapt to the feedback. The absorptive capacity is constructed and the technological innovation capability is improved. Therefore, the latecomer enterprises improve the technological innovation capability in the process of "internalization" "externalization" and "combination" of knowledge. As a good driving mechanism, the knowledge cycle mechanism promotes the "self-evolution" of the coacting system.

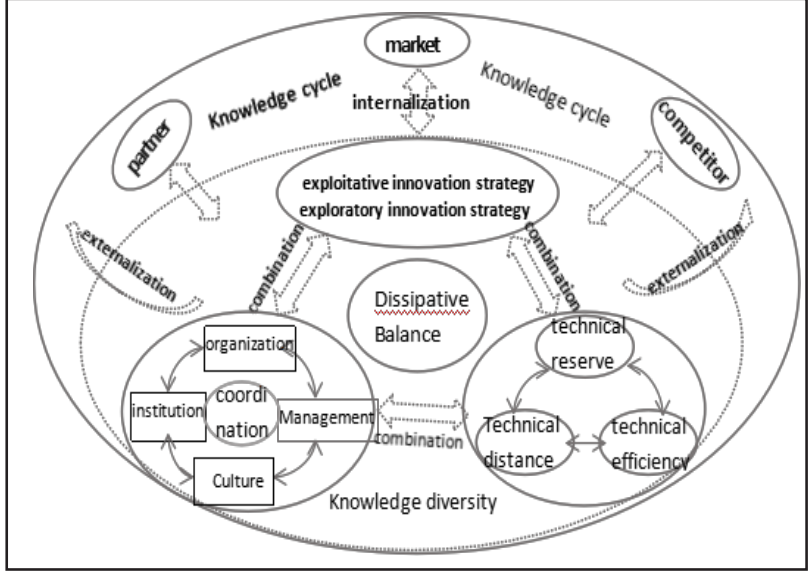

Figure 1. Dynamic evolution model of "Innovation Strategy-Innovation Paradigm-technological Innovation capability".

Second, the principle of "species" diversity: in different stages of technological innovation, enterprises construct different learning mechanisms to absorb heterogeneous knowledge and ensure the "richness" and "complexity" of the enterprise knowledge base. In this way, companies can avoid their own technological rigidity, increase the possibility of breakthrough innovation, and maintain the ecological balance of enterprise knowledge, which is similar to the mechanism of species diversity affecting ecosystem balance. Therefore, the principle of knowledge diversity ensures the "self-evolution" mechanism of the catch-up system of latecomer firms.

Third, Dissipative Structure Theory: the dissipation theory in ecosystem shows that in order to maintain its stability, the system must coordinate the energy input outward and inward to ensure the balance of positive and negative entropy flow. This is a good explanation for the internal reasons of patent cross-licensing and even joint ventures with competitors in the late growth period of latecomer firms: enterprises achieve ecological balance by coordinating the flow rate of resources. Therefore, both enterprise ecology and natural ecology must be in a dynamic and stable state in order to maintain their ability of sustainable development. The excavation and application of dissipation principle is a catalyst for latecomer firms to realize "bend overtaking" and the high speed catch-up.

\section{Conclusions}

Based on the grounded theory, this study measures the innovation strategy, innovation paradigm and technological innovation capability in the process of Huawei's development, and constructs an integrated evolution model.

First, Huawei's technological innovation capability improvement path is "Imitation Innovation capability Primary Secondary Innovation capability - Ripe Secondary Innovation capability - Integrated Innovation capability - Original Innovation capability". Only by mastering the ability to improve the "core structure" of the technological paradigm can enterprises realize the 
"change of track" and get out of the development circle of "backwardness-introduction, backwardness and reintroduction".

Second, The innovation paradigm and its innovation capability have evolved with the innovation strategy. This paper constructs the integrated evolution model among the three, and reveals the internal mechanism of its evolution. After research, it is concluded that strategy leads innovation, innovation paradigm is the support of strategy implementation, Innovation paradigm is the basis of the formation of technological Innovation capability.

Third, By studying the integrated evolution model of the innovation strategy, innovation paradigm and technological innovation capability in the catch-up process of latecomer firms, this paper draws the conclusion that: the driving mechanism, regulating mechanism and catalytic mechanism among the integrated evolution model in SPT of LCFs' are respectively the Knowledge Cycle Mechanism, Knowledge Diversity and Dissipative Structure Theory.

\section{ACKNOWLEDGMENT}

The authors greatly acknowledge the sponsorship provided by National Nature Science Foundation of China(71572177), Chinese Academy of Engineering (2017-XY-39), Tianyou Youth Talent Lift Program of Lanzhou Jiaotong University.

\section{References}

1. J. A. Mathews and D. S. Cho, "Combinative capabilities and organizational learning by latecomer firms:The case of the Korean Semiconductor Industry," vol. 34, 1999, pp.139-156.

2. L. T. Chen, "Rule of enterprise'innovation capability evolution:Based on adaptive evolution and synergy perspectives," Zhejiang University, 2012, pp.197.

3. H. B. Liu and Q. R. Xu, "Latecomer firms strategy evolution,innovation paradigm and capabilities evolution," Beijing, vol. 36, 2018, 1442-54.

4. Q. R. Xu, Z. Y. Wu and L. T. Chen, "An analysis of the evolution path to and the driving factors of the independent innovation of enterprise in the transitional economy," Beijing, 2013, vol.4, pp.121$134+188$.

5. E. Larry, Westphal, W. R. Yung, Garry and H. "Pursell, Sources of Technological Capability in South Area," London: Macmillan, 1984, pp. 163.

6. P. J. Sher and P. Y. Yang, "The Effects of Innovative Capabilities and R\&D Clustering on Firm Performance:The Evidence of Taiwan's Semiconductor Industry," Technovation., 2005, vol. 25, pp.33-43.

7. M. Fransman, "Conceptualizing Technical Change in the Third World in the 1980's:An Interpretive Survey," Journal of Development Studies, vol .21, 1984, pp. 572.

8. G. Qu and X. Liu, "Discussion on Establishing the Evaluation Index System of Enterprise Technical Innovation Capability," Journal of Liaoning Institute of Technology, 1999, vol. 1, pp.79-82.

9. M. Benner and M. Tushman, "Process management and technological innovation:A longitudinal study of the photography and paint industries," Administration Science Quarterly,vol. 47, 2002, pp.676-706.

10. X. Wu, Y. Fu, D. Wu and L. Lei, "How Do Latecomers Transform from Catch-up to Beyond Catch-up?" Beijing, Management World, vol.2, 2019, pp.151-167+200. 